\title{
A Done Delivery Network for Acute Traumatic Brain Injury Management in the Caribbean: A Commentary
}

\author{
Ashley Williams ${ }^{1}$, Myron Rolle ${ }^{2}$
}

\begin{abstract}
Medical products transportation has become an important research topic requiring multidisciplinary collaboration among experts in surgery, public health, and health economics. Drones can be a promising tool to increase access to stabilizing neurotrauma care following a pediatric traumatic brain injury in the Caribbean. This commentary describes how the use of drones and neurotrauma education can increase health outcomes of neurosurgical emergencies on remote islands of low-and middle-income nations in the developing world.

Keywords: Advanced trauma life support care, Brain concussion, Brain trauma, Brain trauma injury, Medical education, Neurosurgery, Neurotrauma, Pediatric trauma, Prehospital care, Trauma nursing.

Panamerican Journal of Trauma, Critical Care \& Emergency Surgery (2021): 10.5005/jp-journals-10030-1361
\end{abstract}

A child in Inagua, one of the many remote family islands in the Bahamas, suffers a life-threatening traumatic brain injury (TBI) after a road traffic accident. She is slipping in and out of consciousness. The local providers who have been trained by the CARICOM Neurosurgical Initiative Virtual Neurotrauma Module-realize quickly that the child's TBI requires acute stabilization within the golden four hour window. ${ }^{1,2}$ Prior to any definitive neurosurgical intervention, the patient could require any of the following: intravenous access, sodium chloride infusion, hyperosmolar solutions, anti-epileptic drugs, antihypertensive drugs, pain medications, sedatives, and blood products. The challenges these local Inaguan providers face, like many Caribbean medical professionals, is (1) a widespread decentralized geography which hinders access and (2) limited resources to provide urgent or emergent care in neurotrauma cases. Thinking creatively about modern medical global technology yields one potential solution to said access and resource challenge: drones.

One may query, what key instrument can help close the gap between acute injury and access to life-sustaining care? A drone. The term "drone" was first used to describe the male bee; yet, in the 1920s it was also introduced for aerial vehicles which hummed similarly to that of a male bee. ${ }^{3}$ While drones have a long history in military use, most interesting is how drones have been implicated in medical transport, especially in low- and middle-income countries. Sub-Saharan Africa is leading the world in medical drone use. ${ }^{4}$ In Rwanda, blood delivery is hindered by poor roads across diverse terrain and transfusion clinics that are not able to store large quantities of blood or predict needed quantities of future blood. In 2016, Rwanda was the first country to become successful at nationally integrating drones into health services; this enabled blood transfusion clinics to treat life-threatening postpartum hemorrhage, the leading cause of death for pregnant women, by placing emergency blood orders by cell phone text message. In about 30 minutes, a Zipline drone can fulfill a clinician's request. As a result, Rwandan blood delivery times to rural areas have decreased from about 4 hours to 15-45 minutes which resulted in over 18,000 lifesaving delivery flights containing blood products were made as of August 2019. ${ }^{5}$ Similar drone programs were implemented in Tanzania and Ghana to deliver malaria
${ }^{1}$ Oakland University William Beaumont School of Medicine, Rochester, Michigan, United States

${ }^{2}$ Program in Global Surgery and Social Change, Harvard Medical School, Boston, Massachusetts, United States

Corresponding Author: Myron Rolle, Program in Global Surgery and Social Change, Harvard Medical School, Boston, Massachusetts, United States of America, e-mail: mrolle@partners.org

How to cite this article: Williams A, Rolle M. A Done Delivery Network for Acute Traumatic Brain Injury Management in the Caribbean: A Commentary. Panam J Trauma Crit Care Emerg Surg 2021;10(3):145-146.

Source of support: Nil

Conflict of interest: None

drugs, antiretrovirals, and blood products. ${ }^{6,7}$ In 2020, Rwanda's established drone program became crucial for disseminating public information about COVID-19. ${ }^{8}$ Evidence of drone use in these realms, suggest that drones can be useful providing acute care for TBI.

For drones to work for remote neurosurgical cases in the Caribbean landscape, a few system items must be in place. The large hospital center in the island must have the available medications and resources in stock to send via drone to the targeted remote location of the TBI patient. The communication between teams has to be efficient and seamless in order to facilitate the delivery of the appropriate resources in a timely fashion. The drones must be managed preflight, intraflight, and postflight by skilled technicians in order to sustain the program for future uses. The receiving providers of the drones' resources must be capable and equipped to put the delivered materials to life-saving use. The receiving providers should also have storage units readily available for any unused resources.

While drones are an effective initial step in TBI management, definitive surgery for compressive lesions in a salvageable patient still remains the gold standard. Thus, concomitantly with drone utilization for stabilization of acute TBI patients in remote islands, the large hospital centers in the island should be preparing for a physical evacuation of that patient to determine if neurosurgical intervention is required. 
An abundance of neurosurgical literature has reported that the prognosis of patients who have reduced intracranial swelling, limited seizure activity, improved Glasgow Coma Scale scores, and controlled blood pressure is much better than those who do not. ${ }^{9,10}$ Drones can significantly help achieve these favorable prognostic factors-essentially saving the life of a patient who otherwise would decompensate quickly.

For the child in Inagua with a TBI, her chance of survival improves with acute stabilization measures. An organized health system, adept personnel and the utility of drones can help save this young patient's life. Major stakeholders, government leaders, and providers should strongly consider the implementation of drones to overcome the inherent challenges in the Caribbean when it comes to managing TBI.

\section{References}

1. Seelig JM, Becker DP, Miller JD, et al. Traumatic acute subdural hematoma: major mortality reduction in comatose patients treated within four hours. N Engl J Med 1981;304(25):1511-1518. DOI: 10.1056/NEJM198106183042503

2. Stiver SI,Manley GT. Prehospital management of traumatic brain injury. Neurosurg Focus 2008;25(4):E5. DOI: 10.3171/FOC.2008.25.10.E5

3. Dekoulis G. Introductory Chapter: Drones. In: Dekoulis G, ed. Drones - Applications. InTech; 2018. DOI: 10.5772/intechopen.76943
4. Holst C, Sukums F, Radovanovic D, et al. Sub-Saharan Africa-the new breeding ground for global digital health. Lancet Digit Health 2020;2(4):e160-e162. DOI: 10.1016/S25897500(20)30027-3

5. Rwanda launches world's first national drone delivery service powered by Zipline. Gavi: The Vaccine Alliance. Accessed July 15, 2021. https://www.gavi.org/news/media-room/rwanda-launchesworlds-first-national-drone-delivery-service-powered-zipline

6. Knott S. In Ghana, Drones Help Combat COVID-19| Voice of America - English. VOA News. Published April 29, 2020. Accessed July 15, 2021. https://www.voanews.com/covid-19-pandemic/ghana-drones-helpcombat-covid-19

7. Vincent J. Self-flying drones are helping speed deliveries of COVID19 vaccines in Ghana. The Verge. Published March 9, 2021. Accessed July 15, 2021. https://www.theverge.com/2021/3/9/22320965/dronedelivery-vaccine-ghana-zipline-cold-chain-storage/

8. Karim N, Jing L, Lee JA, et al. Lessons learned from Rwanda: innovative strategies for prevention and containment of COVID-19. Ann Glob Health 2021;87(1):23. DOI:10.5334/aogh.3172

9. AhI R, Sarani B, Sjolin G, et al. The association of intracranial pressure monitoring and mortality: a propensity score-matched cohort of isolated severe blunt traumatic brain injury. J Emerg Trauma Shock 2019;12(1):18-22. DOI: 10.4103/JETS.JETS_59_18

10. Hemphill 3rd JC, Bonovich DC, Besmertis L, et al. The ICH score: a simple, reliable grading scale for intracerebral hemorrhage. Stroke 2001;32(4):891-897. DOI: 10.1161/01.str.32.4.891 